\title{
Periodic and Spline Multiresolution Analysis and the Lifting Scheme
}

\author{
Jürgen Prestin ${ }^{1}$ and Ewald Quak ${ }^{2}$ \\ 1 Institut für Mathematik Universität zu Lübeck Wallstrasse 40 D-23560 Lübeck \\ Germany prestin@math.uni-luebeck.de \\ 2 Department of Applied Mathematics SINTEF ICT Forskningsveien 1 NO-0314 \\ Oslo Norway Ewald.Quak@sintef .no
}

Summary. The lifting scheme is a well-known general framework for the construction of wavelets, especially in finitedimensional settings. After a short introduction about the basics of lifting, it is discussed how wavelet constructions in two specific finite settings can be related to the lifting approach. These examples concern on one hand polynomial splines and on the other the Fourier approach for translation invariant spaces of periodic functions.

\section{Introduction}

The goal of this contribution is to address how two types of multiresolution analysis that have been subject to research activities within the MINGLE project are related to a well-known general framework for the construction of wavelets, namely the lifting scheme, as introduced by Sweldens [Sw96], see also Sweldens \& Schröder [SchSw96], Daubechies \& Sweldens [DSw98] and the references therein. In the original paper [Sw96] the approach is formulated for the whole real axis, but it can also be used to generate various sorts of "second-generation wavelets" in finite settings or to describe decomposition and reconstruction algorithms without ever explicitly working with basis functions. In our paper, we describe a general finite setting with basis functions and related matrices that nicely fits the specific types of multiresolution we are most interested in, namely polynomial splines and periodic functions, yet we do not aim to address the topic in the full generality given by the concept of stable completions as described by Carnicer, Dahmen \& Pena [CDP96]. Thus we also do not address in any detail the construction of biorthogonal wavelets on the interval as addressed for example in [M96], [DS99], [S98] and [DKU99]. The lifting framework we want to relate to is the topic of Section 2, spline examples are presented in Section 3, and the Fourier approach for periodic functions is covered in Section 4. 


\section{Basics about the Lifting Scheme}

\subsection{Decomposition of a finitedimensional space}

We will be dealing with real-valued square-integrable univariate functions defined over a compact interval and we use for simplicity always the standard inner product $\langle\cdot, \cdot\rangle$. Our setting is strictly finitedimensional, meaning that a linear space of functions $U$ of dimension $n+m$ is represented as the direct sum of two spaces, namely $V^{0}$ of dimension $n$ and $W^{0}$ of dimension $m$, i.e.

$$
U=V^{0}+W^{0} .
$$

Let a basis of $U$ be given by functions $\vartheta_{0}, \ldots, \vartheta_{n+m-1}$, a basis of $V^{0}$ by functions $\varphi_{0}^{0}, \ldots, \varphi_{n-1}^{0}$ and a basis of $W^{0}$ by functions $\psi_{0}^{0}, \ldots, \psi_{m-1}^{0}$. For ease of notation we use column vectors of functions as

$$
\boldsymbol{\vartheta}=\left(\vartheta_{0}, \ldots, \vartheta_{n+m-1}\right)^{T}, \boldsymbol{\varphi}^{0}=\left(\varphi_{0}^{0}, \ldots, \varphi_{n-1}^{0}\right)^{T} \text { and } \boldsymbol{\psi}^{0}=\left(\psi_{0}^{0}, \ldots, \psi_{m-1}^{0}\right)^{T} .
$$

Due to (1), there exist matrices $\mathbf{P}^{0}$ of dimension $(n+m) \times n$ and $\mathbf{Q}^{0}$ of dimension $(n+m) \times m$, such that

$$
\left(\begin{array}{c}
\boldsymbol{\varphi}^{0} \\
\boldsymbol{\psi}^{0}
\end{array}\right)^{T}=\boldsymbol{\vartheta}^{T}\left(\mathbf{P}^{0}, \mathbf{Q}^{0}\right)=\boldsymbol{\vartheta}^{T} \mathbf{M}^{0}
$$

As the functions are chosen as bases of their respective spaces, the two-scale matrix $\mathbf{M}^{0}$ is nonsingular and we write $\left(\mathbf{M}^{0}\right)^{-1}=\left(\tilde{\mathbf{P}}^{0}, \tilde{\mathbf{Q}}^{0}\right)^{T}$, again with matrices $\tilde{\mathbf{P}}^{0}$ of dimension $(n+m) \times n$ and $\tilde{\mathbf{Q}}^{0}$ of dimension $(n+m) \times m$, yielding

$$
\boldsymbol{\vartheta}^{T}=\left(\begin{array}{c}
\boldsymbol{\varphi}^{0} \\
\boldsymbol{\psi}^{0}
\end{array}\right)^{T}\left(\tilde{\mathbf{P}}^{0}, \tilde{\mathbf{Q}}^{0}\right)^{T}
$$

Proposition 1. The fact that the matrices $\mathbf{M}^{0}$ and $\left(\mathbf{M}^{0}\right)^{-1}$ are inverses is reflected in the matrix relations

$$
\left(\begin{array}{c}
\tilde{\mathbf{P}}^{0^{T}} \mathbf{P}^{0}, \tilde{\mathbf{P}}^{0^{T}} \mathbf{Q}^{0} \\
\tilde{\mathbf{Q}}^{0^{T}} \mathbf{P}^{0}, \tilde{\mathbf{Q}}^{0^{T}} \mathbf{Q}^{0}
\end{array}\right)=\left(\begin{array}{cc}
\mathbf{I}_{n} & \mathbf{0}_{n \times m} \\
\mathbf{0}_{m \times n} & \mathbf{I}_{m}
\end{array}\right)
$$

and

$$
\mathbf{P}^{0} \tilde{\mathbf{P}}^{0^{T}}+\mathbf{Q}^{0} \tilde{\mathbf{Q}}^{0^{T}}=\mathbf{I}_{n+m}
$$

An element $h \in U$ can be written as $h=f+g$, where $f \in V^{0}$ and $g \in W^{0}$, and in the respective basis representations as

$$
\boldsymbol{\vartheta}^{T} \mathbf{h}=\boldsymbol{\varphi}^{0^{T}} \mathbf{f}^{0}+\boldsymbol{\psi}^{0^{T}} \mathbf{g}^{0}
$$

with coefficient vectors $\mathbf{f}^{0}, \mathbf{g}^{0}$ and $\mathbf{h}$ of length $n, m$ and $n+m$, respectively. We can derive from (3) and (4) 
Proposition 2. Decomposition of the coefficient vector $\mathbf{h}$ into the coefficient vectors of its components is achieved by

$$
\mathbf{f}^{0}=\tilde{\mathbf{P}}^{0^{T}} \mathbf{h} \text { and } \mathbf{g}^{0}=\tilde{\mathbf{Q}}^{0^{T}} \mathbf{h},
$$

while reconstruction of the coefficient vector $\mathbf{h}$ from the coefficient vectors of its components is carried out by

$$
\mathbf{h}=\mathbf{P}^{0} \mathbf{f}^{0}+\mathbf{Q}^{0} \mathbf{g}^{0}
$$

In the lazy wavelet approach, the spaces $V^{0}$ and $W^{0}$ are generated by just dividing the basis functions into two disjoint groups, typically by separating the even-index ones from the odd-index ones. For the coefficients this means that decomposition is even and odd subsampling, and the two-scale matrices $\mathbf{M}^{0}$ and $\left(\mathbf{M}^{0}\right)^{-1}$ are simply permutation matrices.

A different strategy is a hierarchical basis approach. If $U$ and $V^{0}$ are given together with basis functions that are somehow linked to a coarse knot sequence $\boldsymbol{\tau}$ for $V^{0}$ and a refinement $\mathbf{t}$ of $\boldsymbol{\tau}$ as the knot sequence for $U$, then it is natural to define a complement $W^{0}$ as the span of those basis functions in the large space $U$ that can be associated with the newly added knots in $\mathbf{t} \backslash \boldsymbol{\tau}$. With the matrix $P^{0}$ fixed by the choice of basis functions in $U$ and $V^{0}$, the columns of the two-scale matrix $\mathbf{Q}^{0}$ are then some unit vectors in $\mathbb{R}^{n+m}$, as determined by the positioning of the new knots with respect to the old ones.

Given once more $U$ and $V^{0}$ together with their basis functions, the semiorthogonal approach uses the uniquely determined relative orthogonal complement $W^{\perp}$ of $V^{0}$ with respect to $U$. The term semi indicates that there is orthogonality between spaces, but each basis need not be an orthogonal basis of the corresponding space.

Taking the point of view of filtering discrete data, one is more interested in the properties of the matrices used for decomposition and reconstruction according to Propositions 1 and 2 than in the actual underlying spaces of functions. A general investigation of the matrix relations (3) and (4) concerning banded matrices can be found in [DM93].

\subsection{Change of basis}

We now want to replace the direct sum in (1) by another one, where we still keep the dimensions of the subspaces, such that

$$
U=V+W
$$

with new bases

$$
\boldsymbol{\varphi}=\left(\varphi_{0}, \ldots, \varphi_{n-1}\right)^{T} \text { and } \boldsymbol{\psi}=\left(\psi_{0}, \ldots, \psi_{m-1}\right)^{T}
$$


for $V$ and $W$, respectively. Note that we leave the basis of $U$ unchanged. Let a nonsingular change-of-basis or transfer matrix be given by

$$
\left(\begin{array}{c}
\boldsymbol{\varphi} \\
\boldsymbol{\psi}
\end{array}\right)^{T}=\left(\begin{array}{c}
\boldsymbol{\varphi}^{0} \\
\boldsymbol{\psi}^{0}
\end{array}\right)^{T}\left(\begin{array}{cc}
\mathbf{A}_{n} & \mathbf{B}_{n \times m} \\
\mathbf{C}_{m \times n} & \mathbf{D}_{m}
\end{array}\right) .
$$

Lemma 1. The two-scale matrix in

$$
\left(\begin{array}{c}
\boldsymbol{\varphi} \\
\boldsymbol{\psi}
\end{array}\right)^{T}=\boldsymbol{\vartheta}^{T}(\mathbf{P}, \mathbf{Q})=\boldsymbol{\vartheta}^{T} \mathbf{M}
$$

and the one in (2) are connected by the same change-of-basis matrix as for the basis functions in (6), in the sense that

$$
(\mathbf{P}, \mathbf{Q})=\left(\mathbf{P}^{0}, \mathbf{Q}^{0}\right)\left(\begin{array}{cc}
\mathbf{A}_{n} & \mathbf{B}_{n \times m} \\
\mathbf{C}_{m \times n} & \mathbf{D}_{m}
\end{array}\right) .
$$

Condition (3) implies

$$
(\tilde{\mathbf{P}}, \tilde{\mathbf{Q}})^{T}=\left(\begin{array}{cc}
\mathbf{A}_{n} & \mathbf{B}_{n \times m} \\
\mathbf{C}_{m \times n} & \mathbf{D}_{m}
\end{array}\right)^{-1}\left(\tilde{\mathbf{P}}^{0}, \tilde{\mathbf{Q}}^{0}\right)^{T} .
$$

Special cases for transfer matrices are of course

$$
\left(\begin{array}{cc}
\mathbf{A}_{n} & \mathbf{B}_{n \times m} \\
\mathbf{0}_{m \times n} & \mathbf{D}_{m}
\end{array}\right) \text { and }\left(\begin{array}{cc}
\mathbf{A}_{n} & \mathbf{0}_{n \times m} \\
\mathbf{C}_{m \times n} & \mathbf{D}_{m}
\end{array}\right) .
$$

The first one leaves the space $V^{0}$ intact, and just changes its basis, while the basis $\psi^{0}$ is changed so that the complement space $W^{0}$ changes to (in general a different) $W$. Analogously, the second one leaves the complement space $W^{0}$ intact with a change of basis, and replaces $\varphi^{0}$ and thus the space $V^{0}$ by $V$. Choosing for the first matrix in addition $\mathbf{A}_{n}$ as the identity $\mathbf{I}_{n}$, leaves also the basis of the space $V^{0}$ unchanged, and analogously the choice of $\mathbf{D}_{m}=\mathbf{I}_{m}$ preserves the basis of the complement space $W^{0}$ in the second case.

For what is called lifting, the transfer matrix is even further specialized, as both blocks on the diagonals in (7) are chosen as identities.

Definition 1. A basis $\boldsymbol{\psi}$ for a new complement space $W$ in the decomposition (5) is generated from the old basis $\psi^{0}$ of the old complement space $W^{0}$ in (1) by a lifting step if the change-of-basis matrix has the form

$$
\left(\begin{array}{cc}
\mathbf{I}_{n} & \mathbf{S}_{n \times m} \\
\mathbf{0}_{m \times n} & \mathbf{I}_{m}
\end{array}\right) .
$$

Analogously a basis $\varphi$ for the new space $V$ in the decomposition (5) is generated from the old basis $\varphi^{0}$ of the old space $V^{0}$ in (1) by a dual lifting step if the change-of-basis matrix has the form

$$
\left(\begin{array}{cc}
\mathbf{I}_{n} & \mathbf{0}_{n \times m} \\
\mathbf{S}_{m \times n} & \mathbf{I}_{m}
\end{array}\right) .
$$


The intention of lifting is to change one of the subspaces in (1) by replacing its given and typically "simpler" basis by a new, in some sense "better" basis in a straightforward manner, namely by a suitable choice of the free parameters which are represented by the coefficients in the matrix $\mathbf{S}$. A significant advantage of a basis created by a lifting step is that the inverse of its transfer matrix is very straightforward, namely

$$
\left(\begin{array}{cc}
\mathbf{I}_{n} & \mathbf{S}_{n \times m} \\
\mathbf{0}_{m \times n} & \mathbf{I}_{m}
\end{array}\right)^{-1}=\left(\begin{array}{cc}
\mathbf{I}_{n} & -\mathbf{S}_{n \times m} \\
\mathbf{0}_{m \times n} & \mathbf{I}_{m}
\end{array}\right) .
$$

Using Lemma 1 this implies

Lemma 2. A lifting step changes the decomposition (1) to $U=V^{0}+W$ by performing the change of basis

$$
\left(\begin{array}{c}
\varphi \\
\psi
\end{array}\right)=\left(\begin{array}{c}
\varphi^{0} \\
\psi^{0}+\mathbf{S}_{n \times m}^{T} \varphi^{0}
\end{array}\right)
$$

and the change in two-scale matrices given by

$$
\begin{aligned}
(\mathbf{P}, \mathbf{Q}) & =\left(\mathbf{P}^{0}, \mathbf{Q}^{0}+\mathbf{P}^{0} \mathbf{S}_{n \times m}\right) \\
(\tilde{\mathbf{P}}, \tilde{\mathbf{Q}})^{T} & =\left(\tilde{\mathbf{P}}^{0}-\tilde{\mathbf{Q}}^{0} \mathbf{S}_{n \times m}^{T}, \tilde{\mathbf{Q}}^{0}\right)^{T} .
\end{aligned}
$$

A dual lifting step changes the decomposition (1) to $U=V+W^{0}$ through the change of basis

$$
\left(\begin{array}{c}
\varphi \\
\psi
\end{array}\right)=\left(\begin{array}{c}
\varphi^{0}+\mathbf{S}_{m \times n}^{T} \psi^{0} \\
\psi^{0}
\end{array}\right)
$$

and the change of matrices

$$
\begin{aligned}
(\mathbf{P}, \mathbf{Q}) & =\left(\mathbf{P}^{0}+\mathbf{Q}^{0} \mathbf{S}_{m \times n}, \mathbf{Q}^{0}\right) \\
(\tilde{\mathbf{P}}, \tilde{\mathbf{Q}})^{T} & =\left(\tilde{\mathbf{P}}^{0}, \tilde{\mathbf{Q}}^{0}-\tilde{\mathbf{P}}^{0} \mathbf{S}_{m \times n}^{T}\right)^{T} .
\end{aligned}
$$

In terms of Proposition 2 this means that if the original decomposition matrices $\tilde{\mathbf{P}}^{0}$ and $\tilde{\mathbf{Q}}^{0}$ are sparse and the matrix $\mathbf{S}_{n \times m}$ used to satisfy additional properties is sparse as well, the lifting scheme produces a better basis that still allows efficient computations.

\subsection{The biorthogonal setting}

So far we have only considered one decomposition (1). Since there are two pairs of matrices $(\mathbf{P}, \mathbf{Q})$ and $(\tilde{\mathbf{P}}, \tilde{\mathbf{Q}})$ associated with this decomposition, it is natural to consider a second related decomposition as well. So we will now be looking at two sets of decompositions that are dual to each other. Let 


$$
\begin{aligned}
U & =V^{0}+W^{0} \\
\tilde{U} & =\tilde{V}^{0}+\tilde{W}^{0}
\end{aligned}
$$

with bases for $U, V^{0}, W^{0}$

$\boldsymbol{\vartheta}=\left(\vartheta_{0}, \ldots, \vartheta_{n+m-1}\right)^{T}, \boldsymbol{\varphi}^{0}=\left(\varphi_{0}^{0}, \ldots, \varphi_{n-1}^{0}\right)^{T}$ and $\boldsymbol{\psi}^{0}=\left(\psi_{0}^{0}, \ldots, \psi_{m-1}^{0}\right)^{T}$

and for $\tilde{U}, \tilde{V}^{0}, \tilde{W}^{0}$

$\tilde{\boldsymbol{\vartheta}}=\left(\tilde{\vartheta}_{0}, \ldots, \tilde{\vartheta}_{n+m-1}\right)^{T}, \tilde{\boldsymbol{\varphi}}^{0}=\left(\tilde{\varphi}_{0}^{0}, \ldots, \tilde{\varphi}_{n-1}^{0}\right)^{T}$ and $\tilde{\boldsymbol{\psi}}^{0}=\left(\tilde{\psi}_{0}^{0}, \ldots, \tilde{\psi}_{m-1}^{0}\right)^{T}$

with the corresponding matrices $\mathbf{P}^{0}, \mathbf{Q}^{0}, \tilde{\mathbf{P}}^{0}$ and $\tilde{\mathbf{Q}}^{0}$ given by

$$
\left(\begin{array}{c}
\boldsymbol{\varphi}^{0} \\
\boldsymbol{\psi}^{0}
\end{array}\right)^{T}=\boldsymbol{\vartheta}^{T}\left(\mathbf{P}^{0}, \mathbf{Q}^{0}\right) \text { and }\left(\begin{array}{c}
\tilde{\varphi}^{0} \\
\tilde{\boldsymbol{\psi}}^{0}
\end{array}\right)^{T}=\tilde{\boldsymbol{\vartheta}}^{T}\left(\tilde{\mathbf{P}}^{0}, \tilde{\mathbf{Q}}^{0}\right)
$$

Note that the decomposition $\tilde{U}=\tilde{V}^{0}+\tilde{W}^{0}$ typically involves completely different types of functions than the decomposition $U=V^{0}+W^{0}$. Only the dimensions of the corresponding spaces are supposed to be the same. If in this setting the bases in the large spaces $U$ and $\tilde{U}$ are related by biorthogonality, and the two-scale matrices are inverse transposes, then the bases of the component spaces are dual, as formulated in the following result.

Lemma 3. Let the bases of the large spaces $U$ and $\tilde{U}$ be biorthogonal, i.e.

$$
\langle\boldsymbol{\vartheta}, \tilde{\boldsymbol{\vartheta}}\rangle=\left(\left\langle\vartheta_{i}, \tilde{\vartheta}_{\tilde{\imath}}\right\rangle\right)_{i, \tilde{\imath}=1}^{n+m}=\mathbf{I}_{n+m}
$$

and let the two-scale matrices be related by

$$
\left(\mathbf{P}^{0} \mathbf{Q}^{0}\right)\left(\tilde{\mathbf{P}}^{0} \tilde{\mathbf{Q}}^{0}\right)^{T}=\mathbf{I}_{n+m} .
$$

Then the bases for the subspaces are biorthogonal or dual in the following sense

$$
\left\langle\left(\begin{array}{c}
\boldsymbol{\varphi}^{0} \\
\boldsymbol{\psi}^{0}
\end{array}\right),\left(\begin{array}{c}
\tilde{\boldsymbol{\varphi}}^{0} \\
\tilde{\boldsymbol{\psi}}^{0}
\end{array}\right)\right\rangle=\left(\begin{array}{c}
\left\langle\boldsymbol{\varphi}^{0}, \tilde{\boldsymbol{\varphi}}^{0}\right\rangle\left\langle\boldsymbol{\varphi}^{0}, \tilde{\boldsymbol{\psi}}^{0}\right\rangle \\
\left\langle\boldsymbol{\psi}^{0}, \tilde{\boldsymbol{\varphi}}^{0}\right\rangle\left\langle\boldsymbol{\psi}^{0}, \tilde{\boldsymbol{\psi}}^{0}\right\rangle
\end{array}\right)=\left(\begin{array}{cc}
\mathbf{I}_{n} & \mathbf{0}_{n \times m} \\
\mathbf{0}_{m \times n} & \mathbf{I}_{m}
\end{array}\right) .
$$

In this biorthogonal setting the investigation of a change of bases in the first decomposition must address how the change of bases in the dual spaces must be performed to keep the duality of (11) intact.

Lemma 4. For a nonsingular change-of-basis matrix with

$$
\left(\begin{array}{c}
\boldsymbol{\varphi} \\
\boldsymbol{\psi}
\end{array}\right)^{T}=\left(\begin{array}{c}
\boldsymbol{\varphi}^{0} \\
\boldsymbol{\psi}^{0}
\end{array}\right)^{T}\left(\begin{array}{cc}
\mathbf{A}_{n} & \mathbf{B}_{n \times m} \\
\mathbf{C}_{m \times n} & \mathbf{D}_{m}
\end{array}\right)
$$


the change-of-basis matrix for the dual decomposition must be the inverse transpose to keep the duality intact, i.e.

$$
\left(\begin{array}{c}
\tilde{\varphi} \\
\tilde{\boldsymbol{\psi}}
\end{array}\right)^{T}=\left(\begin{array}{c}
\tilde{\varphi}^{0} \\
\tilde{\boldsymbol{\psi}}^{0}
\end{array}\right)^{T}\left(\begin{array}{cc}
\mathbf{A}_{n} & \mathbf{B}_{n \times m} \\
\mathbf{C}_{m \times n} & \mathbf{D}_{m}
\end{array}\right)^{-T}
$$

results in new, but still dual, decompositions

$$
\begin{aligned}
& U=V+W \\
& \tilde{U}=\tilde{V}+\tilde{W}
\end{aligned}
$$

where the new bases also satisfy

$$
\left(\begin{array}{c}
\langle\boldsymbol{\varphi}, \tilde{\boldsymbol{\varphi}}\rangle\langle\boldsymbol{\varphi}, \tilde{\boldsymbol{\psi}}\rangle \\
\langle\boldsymbol{\psi}, \tilde{\boldsymbol{\varphi}}\rangle\langle\boldsymbol{\psi}, \tilde{\boldsymbol{\psi}}\rangle
\end{array}\right)=\left(\begin{array}{cc}
\mathbf{I}_{n} & \mathbf{0}_{n \times m} \\
\mathbf{0}_{m \times n} & \mathbf{I}_{m}
\end{array}\right)
$$

In the dual setting the simplicity of a lifting step as a change of basis is especially significant. Given that for a lifting step the change-of-basis matrix is

$$
\left(\begin{array}{cc}
\mathbf{I}_{n} & \mathbf{S}_{n \times m} \\
\mathbf{0}_{m \times n} & \mathbf{I}_{m}
\end{array}\right)
$$

its inverse transpose is

$$
\left(\begin{array}{cc}
\mathbf{I}_{n} & \mathbf{0}_{n \times m} \\
-\left(S_{n \times m}\right)^{T} & \mathbf{I}_{m}
\end{array}\right)
$$

meaning that a dual lifting step is performed on the dual decomposition, explaining why the term dual is used in Definition 1.

Corollary 1. A lifting step in $U=V^{0}+W^{0}$ with $\mathbf{S}_{n \times m}$ and a dual lifting step with $-\left(\mathbf{S}_{n \times m}\right)^{T}$ in $\tilde{U}=\tilde{V}^{0}+\tilde{W}^{0}$ result in the dual decompositions

$$
\begin{aligned}
& U=V^{0}+W \\
& \tilde{U}=\tilde{V}+\tilde{W}^{0}
\end{aligned}
$$

with a new pair of dual bases

$$
\left(\begin{array}{c}
\boldsymbol{\varphi} \\
\boldsymbol{\psi}
\end{array}\right)=\left(\begin{array}{c}
\varphi^{0} \\
\boldsymbol{\psi}^{0}+\mathbf{S}_{n \times m}^{T} \boldsymbol{\varphi}^{0}
\end{array}\right) \text { and }\left(\begin{array}{c}
\tilde{\boldsymbol{\varphi}} \\
\tilde{\boldsymbol{\psi}}
\end{array}\right)=\left(\begin{array}{c}
\tilde{\boldsymbol{\varphi}}^{0}-\mathbf{S}_{n \times m} \tilde{\boldsymbol{\psi}}^{0} \\
\tilde{\boldsymbol{\psi}}^{0}
\end{array}\right)
$$

and matrices

$$
\begin{aligned}
(\mathbf{P}, \mathbf{Q}) & =\left(\mathbf{P}^{0}, \mathbf{Q}^{0}+\mathbf{P}^{0} \mathbf{S}_{n \times m}\right) \\
(\tilde{\mathbf{P}}, \tilde{\mathbf{Q}})^{T} & =\left(\tilde{\mathbf{P}}^{0}-\tilde{\mathbf{Q}}^{0} \mathbf{S}_{n \times m}^{T}, \tilde{\mathbf{Q}}^{0}\right)^{T}
\end{aligned}
$$


Corollary 2. A dual lifting step in $U=V^{0}+W^{0}$ with $\mathbf{S}_{m \times n}$ and a lifting step with $-\left(\mathbf{S}_{m \times n}\right)^{T}$ in $\tilde{U}=\tilde{V}^{0}+\tilde{W}^{0}$ generate

$$
\begin{aligned}
U & =V+W^{0} \\
\tilde{U} & =\tilde{V}^{0}+\tilde{W}
\end{aligned}
$$

with a pair of new dual bases

$$
\left(\begin{array}{c}
\boldsymbol{\varphi} \\
\boldsymbol{\psi}
\end{array}\right)=\left(\begin{array}{c}
\varphi^{0}+\mathbf{S}_{m \times n}^{T} \boldsymbol{\psi}^{0} \\
\boldsymbol{\psi}^{0}
\end{array}\right) \text { and }\left(\begin{array}{c}
\tilde{\varphi} \\
\tilde{\boldsymbol{\psi}}
\end{array}\right)=\left(\begin{array}{c}
\tilde{\varphi}^{0} \\
\tilde{\boldsymbol{\psi}}^{0}-\mathbf{S}_{m \times n} \tilde{\boldsymbol{\varphi}}^{0}
\end{array}\right)
$$

and matrices

$$
\begin{aligned}
(\mathbf{P}, \mathbf{Q}) & =\left(\mathbf{P}^{0}+\mathbf{Q}^{0} \mathbf{S}_{m \times n}, \mathbf{Q}^{0}\right) \\
(\tilde{\mathbf{P}}, \tilde{\mathbf{Q}})^{T} & =\left(\tilde{\mathbf{P}}^{0}, \tilde{\mathbf{Q}}^{0}-\tilde{\mathbf{P}}^{0} \mathbf{S}_{m \times n}^{T}\right)^{T} .
\end{aligned}
$$

\subsection{Beyond the original spaces}

So far we have not left the spaces $U$ and $\tilde{U}$, only changed subspaces and their bases. A major point in the real axis setting is that given a biorthogonal setting with finite filters, all other possible pairs with finite filters that are biorthogonal to the original pair can be characterized completely (see Lemma 5 [Sw96]). Such a characterization is of course impossible in a finite setting. According to the lemma below we can, however, say the following. Let $U^{*}$ be another, possibly completely different, large space of functions $U^{*}$ of the correct dimension $n+m$, such that it possesses a basis which is biorthogonal to the basis of $U$. Now we can actually generate a decomposition $V^{*}+W^{*}$ of $U^{*}$ by using the two-scale matrices of $\tilde{U}$ to define the basis functions for the subspaces $V^{*}$ and $W^{*}$. These new basis functions are then still dual to the ones given by the decomposition of $U$.

Lemma 5. Given decompositions $U=V+W$ and $\tilde{U}=\tilde{V}+\tilde{W}$, with biorthogonal bases $\boldsymbol{\vartheta}$ and $\tilde{\boldsymbol{\vartheta}}$ of $U$ and $\tilde{U}$, respectively, let the two-scale matrices be related by

$$
(\mathbf{P} \mathbf{Q})(\tilde{\mathbf{P}} \tilde{\mathbf{Q}})^{T}=\mathbf{I}_{n+m},
$$

so that by Lemma 3

$$
\left(\begin{array}{c}
\langle\boldsymbol{\varphi}, \tilde{\boldsymbol{\varphi}}\rangle\langle\boldsymbol{\varphi}, \tilde{\boldsymbol{\psi}}\rangle \\
\langle\boldsymbol{\psi}, \tilde{\boldsymbol{\varphi}}\rangle\langle\boldsymbol{\psi}, \tilde{\boldsymbol{\psi}}\rangle
\end{array}\right)=\left(\begin{array}{cc}
\mathbf{I}_{n} & \mathbf{0}_{n \times m} \\
\mathbf{0}_{m \times n} & \mathbf{I}_{m}
\end{array}\right) .
$$

Assume that a space $U^{*}$ is spanned by $\boldsymbol{\vartheta}^{*}=\left(\vartheta_{0}^{*}, \ldots, \vartheta_{n+m-1}^{*}\right)^{T}$, where the basis $\boldsymbol{\vartheta}^{*}$ is still biorthogonal to $\boldsymbol{\vartheta}$, i.e. $\left\langle\boldsymbol{\vartheta}, \boldsymbol{\vartheta}^{*}\right\rangle=\mathbf{I}_{n+m}$, and introduce a decomposition $U^{*}=V^{*}+W^{*}$ by defining 


$$
\left(\begin{array}{c}
\boldsymbol{\varphi}^{*} \\
\boldsymbol{\psi}^{*}
\end{array}\right)^{T}:=\boldsymbol{\vartheta}^{*^{T}}(\tilde{\mathbf{P}}, \tilde{\mathbf{Q}})
$$

with $V^{*}$ and $W^{*}$ spanned by $\varphi^{*}$ and $\boldsymbol{\psi}^{*}$, respectively. Then the systems $\varphi^{*}$ and $\boldsymbol{\psi}^{*}$ are also biorthogonal to $\boldsymbol{\varphi}$ and $\boldsymbol{\psi}$ in the sense that

$$
\left(\begin{array}{c}
\left\langle\boldsymbol{\varphi}, \boldsymbol{\varphi}^{*}\right\rangle\left\langle\boldsymbol{\varphi}, \boldsymbol{\psi}^{*}\right\rangle \\
\left\langle\boldsymbol{\psi}, \boldsymbol{\varphi}^{*}\right\rangle\left\langle\boldsymbol{\psi}, \boldsymbol{\psi}^{*}\right\rangle
\end{array}\right)=\left(\begin{array}{cc}
\mathbf{I}_{n} & \mathbf{0}_{n \times m} \\
\mathbf{0}_{m \times n} & \mathbf{I}_{m}
\end{array}\right)
$$

The two-scale matrices for $U^{*}=V^{*}+W^{*}$ remain the same as in (13).

Note that in the classical real axis setting, the basis functions are not just linked within each space by translation, but also the ones in $U$ and $V$ are strongly linked, namely by dilation. In this whole section we have never used such relationships. In the following section, however, we give a short example explaining how one can go beyond the initial spaces in the real axis setting by using that the functions in $U$ and $V$, and $\tilde{U}$ and $\tilde{V}$, respectively, are linked by dilation.

\section{Polynomial splines}

\subsection{Piecewise constants}

We will start by looking at piecewise constant functions, not so much because they are typically used as an example, but because they allow us in fact to investigate a finite setting without too many boundary effects, so that we can go back to an infinite setting of functions on the whole real axis - as we would like to do at the end of this subsection - without too many technical difficulties.

Example 1. Let the interval $[0,1]$ be partitioned by the knots $\tau_{j}=j / 4, j=$ $0, \ldots, 4$. This partition is then uniformly refined to $t_{i}=i / 8, i=0, \ldots, 8$. The space $U$ is the space of piecewise constant functions on the intervals $\left[t_{i}, t_{i+1}\right)$ and the space $V^{0}$ is the space of piecewise constant functions on the intervals $\left[\tau_{j}, \tau_{j+1}\right)$. Denoting by $\chi_{[a, b)}$ the characteristic function of the interval $[a, b)$, the elementary basis functions of $U$ are $\vartheta_{i}=\chi_{\left[t_{i}, t_{i+1}\right)}, i=0, \ldots, 7$, and for $V=V^{0}$ they are $\varphi_{j}=\chi_{\left[\tau_{j}, \tau_{j+1}\right)}, j=0, \ldots, 3$. This fixes the matrix $\mathbf{P}^{0}$ as given in (14).

A hierarchical complement space $W^{0}$ is described by choosing its basis functions to complement the $\varphi_{j}$ 's with those elements from $U$ that correspond exactly to the newly added knots, namely $\psi_{k}^{0}=\vartheta_{2 k+1}, k=0, \ldots, 3$, so that

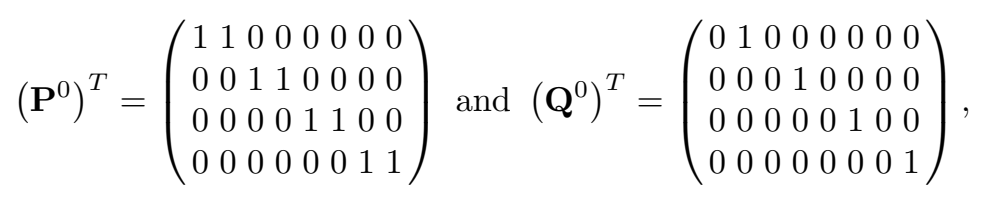


inducing

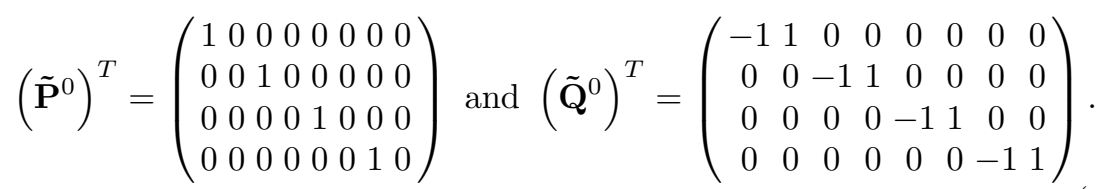

According to Definition 1, a general lifting step matrix in this setting is

$$
\left(\begin{array}{cc}
\mathbf{I}_{4} & \mathbf{S}_{4 \times 4} \\
\mathbf{0}_{4 \times 4} & \mathbf{I}_{4}
\end{array}\right)
$$

where the entries $s_{k, j}$ of $\mathbf{S}_{4 \times 4}$ can still be chosen freely. The old "wavelets" $\boldsymbol{\psi}_{k}^{0}$ are characteristic functions and thus possess no vanishing moments. We now demand that the new basis functions $\psi_{k}$ at least have one vanishing moment (their integrals be zero). This is reflected by the four conditions that $\left\langle\psi_{k}, 1\right\rangle=0$ for all $k$. With a total of 16 coefficients in $\mathbf{S}_{4 \times 4}$, that leaves us with a good deal of free parameters. As we want $S_{4 \times 4}$ to be as sparse as possible, it turns out that a diagonal matrix is sufficient, namely

$$
\mathbf{S}_{4 \times 4}=\left(\begin{array}{cccc}
-\frac{1}{2} & 0 & 0 & 0 \\
0 & -\frac{1}{2} & 0 & 0 \\
0 & 0 & -\frac{1}{2} & 0 \\
0 & 0 & 0 & -\frac{1}{2}
\end{array}\right)
$$

Lemma 2 gives us the new matrices as

$$
\mathbf{Q}^{T}=\left(\begin{array}{cccccccc}
-\frac{1}{2} & \frac{1}{2} & 0 & 0 & 0 & 0 & 0 & 0 \\
0 & 0 & -\frac{1}{2} & \frac{1}{2} & 0 & 0 & 0 & 0 \\
0 & 0 & 0 & 0 & -\frac{1}{2} & \frac{1}{2} & 0 & 0 \\
0 & 0 & 0 & 0 & 0 & 0 & -\frac{1}{2} & \frac{1}{2}
\end{array}\right) \text { and } \tilde{\mathbf{P}}^{T}=\left(\begin{array}{cccccccc}
\frac{1}{2} & \frac{1}{2} & 0 & 0 & 0 & 0 & 0 & 0 \\
0 & 0 & \frac{1}{2} & \frac{1}{2} & 0 & 0 & 0 & 0 \\
0 & 0 & 0 & 0 & \frac{1}{2} & \frac{1}{2} & 0 & 0 \\
0 & 0 & 0 & 0 & 0 & 0 & \frac{1}{2} & \frac{1}{2}
\end{array}\right),
$$

while $\mathbf{P}^{0}$ remains as in (14) and $\tilde{\mathbf{Q}}^{0}$ as in (15).

Consequently in this very special case a lifting step has not just created a new basis (and corresponding complement space) with basis functions that now all possess one vanishing moment, the new functions are in fact the Haar wavelets and the new complement space $W$ is in fact the orthogonal complement $W^{\perp}$, as $\mathbf{S}_{4 \times 4}$ is actually the unique solution to the 16 equations $\left\langle\boldsymbol{\psi}_{k}, \boldsymbol{\varphi}_{j}^{0}\right\rangle=0$ for all $k, j$. That of course is really a special case only valid for piecewise constants, as we will see in the next subsection.

Example 2. In the setting of Example 1, the functions $\vartheta_{i}$ form an orthogonal, but not orthonormal system. It is straightforward to choose a biorthogonal system as $\tilde{\vartheta}_{i}=8 \vartheta_{i}$ for all $i$, so that $\tilde{U}=U$. As matrices we choose $\mathbf{P}^{0}$ from (14), $\tilde{\mathbf{Q}}^{0}$ from (15) and as $\tilde{\mathbf{P}}^{0}$ and $\mathbf{Q}^{0}$ the matrices from (16), as they satisfy the condition (10). Then (8) allows it to compute $\varphi_{j}^{0}$ as used in Example 1, and their duals are $\tilde{\varphi}_{j}^{0}=4 \varphi_{j}^{0}$, with $\psi_{k}^{0}=\frac{1}{2} \vartheta_{2 k+1}-\frac{1}{2} \vartheta_{2 k}$ and $\tilde{\psi}_{k}^{0}=\tilde{\vartheta}_{2 k+1}-\tilde{\vartheta}_{2 k}$. 
In this setting we want to perform a dual lifting step with a matrix $\mathbf{S}_{4 \times 4}$. Let us say that we want to create new dual wavelets $\tilde{\psi}_{k}$ that have three vanishing moments, as the $\tilde{\psi}_{k}^{0}$ 's have only one according to their construction above. For $k=1,2$ there are three free parameters, which we can fix by the conditions for three vanishing moments, i.e. for $e_{i}(x)=x^{i}, i=0,1,2$, we demand that $\left\langle\tilde{\psi}_{k}, e_{i}\right\rangle=0$ for $k=1,2$. A direct computation establishes

$$
\mathbf{S}_{4 \times 4}=\left(\begin{array}{cccc}
s_{0,0} & s_{0,1} & s_{0,2} & 0 \\
-\frac{1}{32} & 0 & \frac{1}{32} & 0 \\
0 & -\frac{1}{32} & 0 & \frac{1}{32} \\
0 & s_{3,1} & s_{3,2} & s_{3,3}
\end{array}\right) .
$$

Note here the boundary effect. If we want the boundary wavelets $\tilde{\psi}_{k}, k=0,3$, to have three vanishing moments as well, we have to use all three parameters, which results in a matrix that is not tridiagonal in the corresponding two corners. If we want a tridiagonal matrix, the boundary functions can only have two vanishing moments at most.

As indicated at the end of the previous section, let us now have a quick look indicating how the lifting scheme on the real axis actually works to define new spaces and new types of functions. To this end choose the matrix in (17) such that we get two diagonals with constant entries, i.e.

$$
\mathbf{S}_{4 \times 4}^{b}=\left(\begin{array}{cccc}
-\frac{1}{32} & \frac{1}{32} & 0 & 0 \\
-\frac{1}{32} & 0 & \frac{1}{32} & 0 \\
0 & -\frac{1}{32} & 0 & \frac{1}{32} \\
0 & 0 & -\frac{1}{32} & \frac{1}{32}
\end{array}\right)
$$

That way the boundary dual wavelets only have one vanishing moment, but that is not so important. The main point, however, is the expression we can derive from (12) for the new inner scaling functions, which is in this case completely free of boundary effects. We obtain for $i=1$ (for $i=2$ this works just as well)

$$
\varphi_{1}=\varphi_{1}^{0}-\frac{1}{32} \psi_{0}^{0}+\frac{1}{32} \psi_{2}^{0} .
$$

In terms of the fine basis of $U$ that means

$$
\varphi_{1}=-\frac{1}{32}\left(\frac{1}{2} \vartheta_{1}-\frac{1}{2} \vartheta_{2}\right)+\vartheta_{3}+\vartheta_{4}+\frac{1}{32}\left(\frac{1}{2} \vartheta_{5}-\frac{1}{2} \vartheta_{6}\right) .
$$

Now recall that in the real axis setting the fine functions are linked to the coarse ones through dilation. Thus (18) can be re-interpreted as a refinement equation in the following sense

$$
\begin{aligned}
\varphi(x)= & -\frac{1}{32}\left(\frac{1}{2} \varphi(2 x+2)-\frac{1}{2} \varphi(2 x+1)\right)+\varphi(2 x)+\varphi(2 x-1) \\
& +\frac{1}{32}\left(\frac{1}{2} \varphi(2 x-2)-\frac{1}{2} \varphi(2 x-3)\right)
\end{aligned}
$$


which defines a completely new scaling function, which is no longer piecewise constant of course. The corresponding new wavelet is given by

$$
\psi(x)=-\frac{1}{2} \varphi(2 x)+\frac{1}{2} \varphi(2 x-1) .
$$

According to the construction, the functions are biorthogonal to the basis pair corresponding on the real axis to $\tilde{\varphi}=\tilde{\varphi}^{0}$ and $\tilde{\psi}=\tilde{\psi}^{0}-S_{4 \times 4} \tilde{\varphi}^{0}$, which are still piecewise constant. As $\tilde{\psi}$ was constructed to have three vanishing moments, the new function $\varphi$ reproduces polynomials of order three.

The functions $\varphi$ and $\psi$ are of course well-known. The lifting scheme produced here the biorthogonal pairs originally constructed by Cohen, Daubechies and Feauveau [CDF92]. In fact a general result of Daubechies and Sweldens [DSw98] shows how on the real axis any biorthogonal pairs with finite filters (finitely many two-scale coefficients) can be factored into lifting steps.

\subsection{Piecewise linear splines}

Given the space constraints of this paper, we outline quickly, just using piecewise linear splines, how higher order splines behave differently to the simple piecewise constants of the previous subsection.

Example 3. We investigate the same knot sequences as in Example 2. This time, however, we consider all piecewise linear functions with respect to the knots $t_{i}$ as forming the space $U$, and all piecewise linear functions with respect to the knots $\tau_{j}$ as forming the subspace $V$. As basis functions of these spaces we consider the "hat" functions (piecewise linear B-splines), that are 1 in one knot and 0 in all others. We denote by $\Lambda_{t_{i}}, i=0, \ldots, 8$, the fine hats for the knots $t_{i}$, and by $\lambda_{\tau_{j}}, j=0, \ldots, 4$, the coarse hats for the knots $\tau_{j}$. Thus $\vartheta_{i}=\Lambda_{t_{i}}$ and $\varphi_{j}=\lambda_{\tau_{j}}$. Again a hierarchical choice for a basis of a complement space $W^{0}$ is fairly obvious: the fine hat functions corresponding to the newly inserted knots. So we set $\psi_{k}^{0}=\vartheta_{2 k+1}=\Lambda_{t_{2 k+1}}, k=0, \ldots, 3$. This is also called the Faber decomposition, see the tutorial [Q02]. The matrices $\mathbf{P}^{0}$ and $\mathbf{Q}^{0}$ are also given explicitly there.

In this case the lifting matrix assumes the form

$$
\left(\begin{array}{cc}
\mathbf{I}_{5} & \mathbf{S}_{5 \times 4} \\
\mathbf{0}_{4 \times 5} & \mathbf{I}_{4}
\end{array}\right),
$$

where the entries $s_{k, j}$ of $\mathbf{S}_{5 \times 4}$ can still be chosen freely.

The basis elements of $W^{0}$ have no vanishing moments at all. So again we can use the free parameters that are provided through $S_{5 \times 4}$ to generate a new basis $\psi_{k}^{1}$ for a new complement $W^{1}$, so that the new functions have vanishing moments. For piecewise linear functions we thus try $\left\langle\psi_{k}^{1}, e_{0}\right\rangle=$ $\left\langle\psi_{k}^{1}, e_{1}\right\rangle=0$ for all $k$. On the other hand we would like to have only a few non-zero coefficients. It turns out that it is possible to satisfy the conditions 
by only involving the two coarse hats neighbouring the fine hat $\psi_{k}^{0}$. In fact the resulting inner functions $\psi_{k}^{1}$ are symmetric, namely

$$
\mathbf{S}_{5 \times 4}^{T}=\left(\begin{array}{ccccc}
-\frac{3}{4} & -\frac{1}{8} & 0 & 0 & 0 \\
0 & -\frac{1}{4} & -\frac{1}{4} & 0 & 0 \\
0 & 0 & -\frac{1}{4} & -\frac{1}{4} & 0 \\
0 & 0 & 0 & -\frac{1}{8} & -\frac{3}{4}
\end{array}\right) \text {. }
$$

Also for linear (and higher order) splines it is then possible to pursue lifting in a biorthogonal setting, producing Cohen-Daubechies-Feauveau pairs on the real axis, but we will not pursue that any further here.

Instead we would like to see how a new basis $\boldsymbol{\psi}_{k}^{\perp}$ spanning the orthogonal complement $W^{\perp}$ of $V$ relative to $U$ can be found by performing a lifting of the Faber basis. Recall that in Example 2, this was already achieved by simply satisfying the condition of one vanishing moment. The analogous matrix (19), however, is not sufficient. The condition of orthogonality of $V$ and $W^{\perp}$ leads to 20 conditions $\left\langle\psi_{k}^{\perp}, \varphi_{j}^{0}\right\rangle=0$, which determine uniquely that

$$
\left(\mathbf{S}_{5 \times 4}^{\perp}\right)^{T}=\frac{1}{224}\left(\begin{array}{ccccc}
-142 & -52 & 14 & -4 & 2 \\
38 & -76 & -70 & 20 & -10 \\
-10 & 20 & -70 & -76 & 38 \\
2 & -4 & 14 & -52 & -142
\end{array}\right) .
$$

Consequently, the matrix $\mathbf{Q}=\mathbf{Q}^{0}+\mathbf{P}^{0} \mathbf{S}_{5 \times 4}^{\perp}$ is dense (see again [Q02]). We conclude that the basis $\psi^{\perp}$ of the orthogonal complement space generated by lifting the Faber basis is the one obtained by solving the normal equations for the fine hats $\Lambda_{t_{2 k+1}}$ (similar results hold for higher order splines).

Thus the following question arises: what is then the change-of-basis matrix that produces the minimally supported B-wavelet basis ([Q02], Lyche, Mørken, [LMQ01]) for $W^{\perp}$ from the hierarchical basis of the Faber decomposition? It is

$$
\left(\begin{array}{cc}
\mathbf{I}_{5} & \mathbf{B}_{5 \times 4} \\
\mathbf{0}_{4 \times 5} & \mathbf{D}_{4}
\end{array}\right)
$$

where

$$
\mathbf{B}_{5 \times 4}^{T}=\left(\begin{array}{ccccc}
-12 & -6 & 0 & 0 & 0 \\
0 & -6 & -6 & 0 & 0 \\
0 & 0 & -6 & -6 & 0 \\
0 & 0 & 0 & -6 & -12
\end{array}\right) \text { and } \mathbf{D}_{4}=\left(\begin{array}{cccc}
20 & 4 & 0 & 0 \\
4 & 16 & 4 & 0 \\
0 & 4 & 16 & 4 \\
0 & 0 & 4 & 20
\end{array}\right),
$$

which is not the matrix of a lifting step, since $D_{4}$ is not the identity. Again similar results using banded matrices, but involving a larger number of bands, hold also for higher order spline B-wavelets. Note finally that the B-wavelets resulting from this change of basis have the same support as the functions obtained through lifting with (19). 
As a concluding remark let us emphasize that in this short treatment of some spline examples, we have obviously downplayed the effect of boundaries and nonuniform knot sequences. A look at [LMQ01] clearly shows that the construction of semi-orthogonal B-wavelets for nonuniform knots involves quite some technical effort. The boundary effects when restricting to an interval the biorthogonal pairs of Cohen, Daubechies and Feauveau, where one component consists of splines over uniform dyadic knots, require also a really substantial treatment, as given by Dahmen, Kunoth and Urban in [DKU99], based on the stable completions of [CDP96].

\section{Periodic Functions}

As a further example we study translation invariant spaces of $2 \pi$-periodic functions. We are again interested in the change of basis algorithms as described in Section 2. Contrary to Section 3, where the transformation matrices in space domain were discussed, it is advantageous in this case to go to the Fourier domain.

Basic results for this approach are studied by Chui \& Mhaskar [CM93], Koh, Lee \& Tan [KLT95], Plonka \& Tasche [PT94],[PT95], Narcowich \& Ward [NW96] and Selig [Se98]. Concerning notations and basic results we follow mainly [Se98]. A matrix formulation of algorithms can also be found in [PQ95]. To begin with let the Fourier matrix $\mathbf{F}$ of dimension $N \times N$ be defined as

$$
\mathbf{F}=\mathbf{F}_{N}=\frac{1}{\sqrt{N}}\left(\mathrm{e}^{-\frac{2 \pi i k l}{N}}\right)_{k, l=0}^{N-1} .
$$

The discrete Fourier transform of length $N$ maps a vector $\mathbf{a}=\left(a_{k}\right)_{k=0}^{N-1} \in \mathbb{C}^{N}$ to a vector $\hat{\mathbf{a}}=\left(\hat{a}_{l}\right)_{l=0}^{N-1}$ by

$$
\hat{a_{l}}=\sum_{k=0}^{N-1} a_{k} \mathrm{e}^{-\frac{2 \pi \mathrm{i} k l}{N}}, \quad l=0, \ldots, N-1,
$$

which can be rewritten as $\hat{\mathbf{a}}=\sqrt{N} \mathbf{F a}$. From the unitarity and symmetry of the Fourier matrix $\mathbf{F}^{-1}=\overline{\mathbf{F}}^{T}=\overline{\mathbf{F}}$ one concludes

$$
\mathbf{a}=\frac{1}{\sqrt{N}} \overline{\mathbf{F}} \hat{\mathbf{a}} \text {. }
$$

Strongly related to the Fourier matrix $\mathbf{F}$ is the decomposition of an arbitrary circulant matrix

$$
\operatorname{circ} \mathbf{a}=\left(a_{(s-r) \bmod N}\right)_{r, s=0}^{N-1}
$$

given by a vector $\mathbf{a} \in \mathbb{C}^{N}$. It holds

$$
\operatorname{circ} \mathbf{a}=\mathbf{F} \operatorname{diag} \hat{\mathbf{a}} \overline{\mathbf{F}},
$$


where $\hat{a}_{l}, l=0, \ldots, N-1$, turn out to be the eigenvalues of the circulant matrix circ a. To introduce the particular function space we have in mind we recall the definition of $L_{2 \pi}^{2}$, the Hilbert space of $2 \pi$-periodic functions with inner product

$$
<\varphi, \psi>=\frac{1}{2 \pi} \int_{0}^{2 \pi} \varphi(t) \overline{\psi(t)} d t
$$

and finite norm $\|\varphi\|^{2}=\langle\varphi, \varphi\rangle$. Every function $\varphi \in L_{2 \pi}^{2}$ can be written as its Fourier series

$$
\varphi(t)=\sum_{k \in \mathbb{Z}} c_{k}(\varphi) \mathrm{e}^{\mathrm{i} k t}
$$

with Fourier coefficients

$$
c_{k}(\varphi)=<\varphi, \mathrm{e}^{\mathrm{i} k \cdot}>=\frac{1}{2 \pi} \int_{0}^{2 \pi} \varphi(t) \mathrm{e}^{-\mathrm{i} k t} d t .
$$

Here we will exploit extensively the relationship to the Hilbert space $\ell^{2}(\mathbb{Z})$ of all sequences $\mathbf{a}=\left(a_{k}\right)_{k \in \mathbb{Z}}$ with

$$
\|\mathbf{a}\|_{\ell^{2}}=\left(\sum_{k \in \mathbb{Z}}\left|a_{k}\right|^{2}\right)^{1 / 2}
$$

and

$$
<\mathbf{a}, \mathbf{b}>_{\ell^{2}}=\sum_{k \in \mathbb{Z}} a_{k} \overline{b_{k}} .
$$

The isomorphism between $L_{2 \pi}^{2}$ and $\ell^{2}(\mathbb{Z})$ is represented by Parseval's equation

$$
\|\varphi\|^{2}=\sum_{k \in \mathbb{Z}}\left|c_{k}(\varphi)\right|^{2} \quad \text { for all } \quad \varphi \in L_{2 \pi}^{2}
$$

and more generally

$$
<\varphi, \psi>=\sum_{k \in \mathbb{Z}} c_{k}(\varphi) \overline{c_{k}(\psi)} \quad \text { for all } \varphi, \psi \in L_{2 \pi}^{2} .
$$

Since we are interested in finite dimensional translation invariant subspaces we define for arbitrary sets of functions $\left\{\varphi_{k}: k=0, \ldots, N-1\right\}, \varphi_{k} \in L_{2 \pi}^{2}$ the Gram matrix

$$
\mathbf{G}=\mathbf{G}(\boldsymbol{\varphi})=\left(<\varphi_{k}, \varphi_{l}>\right)_{k, l=0}^{N-1}
$$

Here and in the following we use the notation $\varphi$ for the vector of functions

$$
\varphi=\left(\varphi_{k}\right)_{k=0}^{N-1} .
$$

We recall for $\mathbf{a}=\left(a_{k}\right)_{k=0}^{N-1} \in \mathbb{C}^{N}$ the simple fact 


$$
\overline{\mathbf{a}}^{T} \mathbf{G a}=\sum_{k=0}^{N-1} \sum_{l=0}^{N-1} \bar{a}_{k}<\varphi_{k}, \varphi_{l}>a_{l}=\left\|\sum_{k=0}^{N-1} \bar{a}_{k} \varphi_{k}\right\|^{2} \geq 0 .
$$

Hence, it follows that $\mathbf{G}$ is regular iff the functions $\varphi_{k}$ are linearly independent. Moreover, if $\mathbf{G}=\mathbf{I}=\mathbf{I}_{N}$ is the identity matrix, then the functions $\varphi_{k}$ are orthonormal. If $\mathbf{G}$ is regular, then

$$
V=\operatorname{span}\left\{\varphi_{k}: k=0, \ldots, N-1\right\} \subset L_{2 \pi}^{2}
$$

is a space of dimension $N$ with basis $\left\{\varphi_{k}: k=0, \ldots, N-1\right\}$. It is now a simple observation that for the basis $\left\{\varphi_{k}\right\}$ there exists a unique biorthogonal basis $\left\{\tilde{\varphi_{k}}: k=0, \ldots, N-1\right\}$ for the space $V$, i.e.,

$$
\left(<\varphi_{k}, \tilde{\varphi}_{l}>\right)_{k, l=0}^{N-1}=\mathbf{I}
$$

is the identity matrix. Furthermore, it holds

$$
\tilde{\varphi}=\mathbf{G}^{-1} \varphi
$$

More generally, every vector of functions $\tilde{\varphi}=\left(\tilde{\varphi}_{l}\right)_{l=0}^{N-1}$ satisfying (20), but not necessarily in $V$ will be called biorthogonal vector for $\varphi=\left(\varphi_{k}\right)_{k=0}^{N-1}$.

From now on we restrict ourselves to finite dimensional translation invariant spaces

$$
V=\operatorname{span}\left\{\varphi\left(\circ-\frac{2 \pi k}{N}\right): k=0, \ldots, N-1\right\} \subset L_{2 \pi}^{2}
$$

generated by a function $\varphi \in L_{2 \pi}^{2}$.

To understand the structure of bases of these spaces we take into account so-called orthogonal splines (cf. [KLT95])

$$
\sum_{p \in \mathbb{Z}} c_{k+N p}(\varphi) \mathrm{e}^{\mathrm{i}(k+N p) \circ}, \quad k=0, \ldots, N-1 .
$$

These orthogonal splines generated from $\varphi \in L_{2 \pi}^{2}$ are related to the translation invariant space $V$ by the following result.

Lemma 6. For $\varphi \in L_{2 \pi}^{2}$ it holds

$$
\begin{aligned}
& \operatorname{span}\left\{\varphi\left(\circ-\frac{2 \pi k}{N}\right): k=0, \ldots, N-1\right\} \\
& =\operatorname{span}\left\{\sum_{p \in \mathbb{Z}} c_{k+N p}(\varphi) \mathrm{e}^{\mathrm{i}(k+N p) \circ}: k=0, \ldots, N-1\right\} .
\end{aligned}
$$

Proof. To show this equality we simply remark

$$
\boldsymbol{\varphi}=\sqrt{N} \mathbf{F}\left(\sum_{p \in \mathbb{Z}} c_{k+N p}(\varphi) \mathrm{e}^{\mathrm{i}(k+N p) \circ}\right)_{k=0}^{N-1},
$$

which can be verified by direct calculation. 
As an immediate consequence of this equality we can compute the dimension of the space $V$ from the sequence of Fourier coefficients $\left(c_{k}(\varphi)\right)$ of its generating function $\varphi$.

Corollary 3. Let $\varphi \in L_{2 \pi}^{2}$. The dimension $d$ of

$$
V=\operatorname{span}\left\{\varphi\left(\circ-\frac{2 \pi k}{N}\right): k=0, \ldots, N-1\right\}
$$

is given by the number of $k, 0 \leq k \leq N-1$, such that there exists a $p \in \mathbb{Z}$ with $c_{k+N p}(\varphi) \neq 0$.

In other words

$$
d=\sharp\left\{k: \sum_{p \in \mathbb{Z}}\left|c_{k+N p}(\varphi)\right|^{2}>0, k=0, \ldots, N-1\right\} .
$$

Particularly, the space $V$ has maximal dimension $N$ iff for all $k$ the orthogonal splines are not the zero function.

Proof. By Parseval's equation the nonzero orthogonal splines are linearly independent. Then the assertion follows immediately from (21).

In the next lemma we discuss under which conditions a function $\psi \in L_{2 \pi}^{2}$ is an element of the subspace $V$.

Lemma 7. The function $\psi \in L_{2 \pi}^{2}$ is an element of $V=\operatorname{span}\left\{\varphi\left(\circ-\frac{2 k \pi}{N}\right)\right.$ : $k=0, \ldots, N-1\}$ iff there exists a vector $\mathbf{a}=\left(a_{s}\right)_{s=0}^{N-1}$ such that

$$
c_{k+N p}(\psi)=\hat{a_{k}} c_{k+N p}(\varphi) \quad \text { for all } \quad k=0, \ldots, N-1 \text { and } p \in \mathbb{Z} .
$$

Then

$$
\psi=\sum_{s=0}^{N-1} a_{s} \varphi\left(\circ-\frac{2 s \pi}{N}\right)
$$

Proof. If (22) is satisfied, then $\psi \in L_{2 \pi}^{2}$ can be written as

$$
\begin{aligned}
\psi(t) & =\sum_{k=0}^{N-1} \hat{a_{k}} \sum_{p \in \mathbb{Z}} c_{k+N p}(\varphi) \mathrm{e}^{\mathrm{i}(k+N p) t} \\
& =\sum_{k=0}^{N-1} \sum_{s=0}^{N-1} a_{s} \mathrm{e}^{-\frac{2 \pi i k s}{N}} \sum_{p \in \mathbb{Z}} c_{k+N p}(\varphi) \mathrm{e}^{\mathrm{i}(k+N p) t} \\
& =\sum_{s=0}^{N-1} a_{s} \varphi\left(t-\frac{2 \pi s}{N}\right)
\end{aligned}
$$

The reverse direction follows in the same way. 
From now on we consider different translation invariant spaces. The first result describes the direct sum property.

Lemma 8. Let $\varphi, \psi \in L_{2 \pi}^{2}$ be given and let

$$
\begin{aligned}
& V=\operatorname{span}\left\{\varphi\left(\circ-\frac{2 k \pi}{N}\right), \quad k=0, \ldots, N-1\right\}, \\
& W=\operatorname{span}\left\{\psi\left(\circ-\frac{2 k \pi}{N}\right), \quad k=0, \ldots, N-1\right\} .
\end{aligned}
$$

Then the following two propositions are equivalent:

I. The sum $V+W$ is a direct one, i.e.

$$
\operatorname{dim} V+\operatorname{dim} W=\operatorname{dim}(V+W) .
$$

II. If there exist $\left(\hat{a_{k}}\right)_{k=0}^{N-1},\left(\hat{b_{k}}\right)_{k=0}^{N-1} \in \mathbb{C}^{N}$ such that

$$
\hat{a_{k}} c_{k+N p}(\varphi)=\hat{b_{k}} c_{k+N p}(\psi) \quad \text { for all } \quad k=0, \ldots, N-1, p \in \mathbb{Z},
$$

$$
\text { then }\left(\hat{a_{k}}\right)=\left(\hat{b_{k}}\right)=\mathbf{0} \text {. }
$$

Proof. We follow the same lines as in the proof of Lemma 7. Writing

$$
\sum_{s=0}^{N-1} a_{s} \varphi\left(\circ-\frac{2 s \pi}{N}\right)-b_{s} \psi\left(\circ-\frac{2 s \pi}{N}\right)=0
$$

in terms of Fourier coefficients gives equivalently

$$
\sum_{s=0}^{N-1} a_{s} \mathrm{e}^{-\frac{2 \pi i \ell s}{N}} c_{\ell}(\varphi)-b_{s} \mathrm{e}^{-\frac{2 \pi i \ell s}{N}} c_{\ell}(\psi)=0 \quad \text { for all } \quad \ell \in \mathbb{Z}
$$

which can be easily reformulated as (25).

The next step is to discuss orthogonality and biorthogonality.

Lemma 9. Let $\varphi, \psi \in L_{2 \pi}^{2}$ be given and let $V$ and $W$ be as in Lemma 8. Then a) $V \perp W$ iff

$$
\sum_{p \in \mathbb{Z}} c_{q+N p}(\varphi) \overline{c_{q+N p}(\psi)}=0 \quad \text { for all } \quad q=0, \ldots, N-1 .
$$

b) The vector of translates $\boldsymbol{\psi}=\left(\psi\left(\cdot-\frac{2 k \pi}{N}\right)\right)_{k=0}^{N-1}$ is a biorthogonal vector for $\boldsymbol{\varphi}=\left(\varphi\left(\cdot-\frac{2 k \pi}{N}\right)\right)_{k=0}^{N-1}$ iff

$$
\sum_{p \in \mathbb{Z}} c_{q+N p}(\varphi) \overline{c_{q+N p}(\psi)}=\frac{1}{N} \quad \text { for all } \quad q=0, \ldots, N-1 .
$$


Proof. Computing for all $0 \leq k, l \leq N-1$ the inner product between the translates of $\varphi$ and $\psi$ we obtain

$$
\begin{aligned}
& \left.\begin{array}{l}
0 \\
\delta_{k, l}
\end{array}\right\}=\left\langle\varphi\left(\circ-\frac{2 k \pi}{N}\right), \psi\left(\circ-\frac{2 l \pi}{N}\right)\right\rangle \\
& =\sum_{s \in \mathbb{Z}} c_{s}(\varphi) \overline{c_{s}(\psi)} \mathrm{e}^{\frac{2 \pi \mathrm{i} s(k-l)}{N}} \\
& =\sum_{q=0}^{N-1} \sum_{p \in \mathbb{Z}} c_{q+N p}(\varphi) \overline{c_{q+N p}(\psi)} \mathrm{e}^{\frac{2 \pi \mathrm{i} q(k-l)}{N}} .
\end{aligned}
$$

Applying the inverse discrete Fourier transform of length $N$ gives the desired results.

Now we are interested in the reverse direction. Let a function $\vartheta \in L_{2 \pi}^{2}$ and a space $U$ be given as

$$
U=\operatorname{span}\left\{\vartheta\left(\circ-\frac{k \pi}{N}\right): k=0, \ldots, 2 N-1\right\} .
$$

Furthermore, we assume $\operatorname{dim} U=2 N$ which means that none of the $2 N$ orthogonal splines generated by $\vartheta$ is the zero function. Now we want to decompose $U$ into the direct sum of translation invariant spaces $V$ and $W$ of the form (23)-(24), i.e.

$$
U=V+W \quad \text { with } \quad \operatorname{dim} V=\operatorname{dim} W=N .
$$

Hence,

$$
\varphi=\sum_{s=0}^{2 N-1} \alpha_{s} \vartheta\left(0-\frac{s \pi}{N}\right), \quad \psi=\sum_{s=0}^{2 N-1} \beta_{s} \vartheta\left(0-\frac{s \pi}{N}\right) .
$$

Under these assumptions one verifies

$$
\hat{\alpha_{k}} \hat{\beta}_{k+N} \neq \hat{\alpha}_{k+N} \hat{\beta}_{k} \quad \text { for all } \quad k=0, \ldots, N-1,
$$

where $\left(\hat{\alpha}_{k}\right)_{k=0}^{2 N-1},\left(\hat{\beta}_{k}\right)_{k=0}^{2 N-1}$ are the discrete Fourier transforms of length $2 N$ of the vectors $\left(\alpha_{s}\right)_{s=0}^{2 N-1}$ and $\left(\beta_{s}\right)_{s=0}^{2 N-1}$, respectively. Note that one can prove the existence of such functions $\varphi, \psi$ starting from an arbitrary $\vartheta \in L_{2 \pi}^{2}$ with $\operatorname{dim} U=2 N$. Note on the other hand that the converse of this statement is not true in general (cf. [Se98], where an example of two orthogonal translation invariant spaces $V, W$ is given for which $U=V+W$ is not a translation invariant space). Furthermore, in [Se98] it is studied how one can describe the basis transformation algorithms in case of $V \perp W$. Here we generalize to the case of a direct sum $V+W$.

Theorem 1. Let the spaces $V$ and $W$ be defined as in Lemma 8. Moreover, let $\operatorname{dim} V+W=2 N$. Then, the basis transformation between 


$$
\vartheta=\left(\vartheta\left(\circ-\frac{s \pi}{N}\right)\right)_{s=0}^{2 N-1} \quad \text { and } \quad\left(\begin{array}{c}
\varphi \\
\psi
\end{array}\right)=\left(\begin{array}{c}
\left(\varphi\left(\circ-\frac{2 s \pi}{N}\right)\right)_{s=0}^{N-1} \\
\left(\psi\left(\circ-\frac{2 s \pi}{N}\right)\right)_{s=0}^{N-1}
\end{array}\right)
$$

can be described easily in the Fourier domain as follows

$$
\left(\begin{array}{c|c}
\overline{\mathbf{F}}_{N} \boldsymbol{\varphi} \\
\overline{\mathbf{F}}_{N} \boldsymbol{\psi}
\end{array}\right)=\frac{1}{\sqrt{2}}\left(\begin{array}{ll}
\operatorname{diag}\left(\hat{\alpha}_{k}\right)_{k=0}^{N-1} & \operatorname{diag}\left(\hat{\alpha}_{k+N}\right)_{k=0}^{N-1} \\
\hline \operatorname{diag}\left(\hat{\beta}_{k}\right)_{k=0}^{N-1} & \operatorname{diag}\left(\hat{\beta}_{k+N}\right)_{k=0}^{N-1}
\end{array}\right) \overline{\mathbf{F}}_{2 N} \boldsymbol{\vartheta}
$$

The inverse formula reads as

$$
\begin{aligned}
\overline{\mathbf{F}}_{2 N} \boldsymbol{\vartheta}= & \sqrt{2}\left(\begin{array}{l|l}
\operatorname{diag}\left(\frac{\hat{\beta}_{k+N}}{\hat{\alpha}_{k} \hat{\beta}_{k+N}-\hat{\alpha}_{k+N} \hat{\beta}_{k}}\right)_{k=0}^{N-1} & \operatorname{diag}\left(\frac{-\hat{\alpha}_{k+N}}{\hat{\alpha}_{k} \hat{\beta}_{k+N}-\hat{\alpha}_{k+N} \hat{\beta}_{k}}\right)_{k=0}^{N-1} \\
\hline \operatorname{diag}\left(\frac{-\hat{\beta}_{k}}{\hat{\alpha}_{k} \hat{\beta}_{k+N}-\hat{\alpha}_{k+N} \hat{\beta}_{k}}\right)_{k=0}^{N-1} & \operatorname{diag}\left(\frac{\hat{\alpha}_{k}}{\hat{\alpha}_{k} \hat{\beta}_{k+N}-\hat{\alpha}_{k+N} \hat{\beta}_{k}}\right)_{k=0}^{N-1}
\end{array}\right) \\
& \times\left(\begin{array}{c}
\overline{\mathbf{F}}_{N} \boldsymbol{\varphi} \\
\overline{\mathbf{F}}_{N} \boldsymbol{\psi}
\end{array}\right) .
\end{aligned}
$$

Proof. With (26) we conclude

$$
\left(\begin{array}{l}
\boldsymbol{\varphi} \\
\boldsymbol{\psi}
\end{array}\right)=\left(\begin{array}{l}
\left(\alpha_{s-2 k}\right)_{k=0, s=0}^{N-1,2 N-1} \\
\left(\beta_{s-2 k}\right)_{k=0, s=0}^{N-1,2 N-1}
\end{array}\right) \boldsymbol{\vartheta}
$$

where we extended the vectors $\left(\alpha_{k}\right)$ and $\left(\beta_{k}\right)$ to be $2 N$-periodic. Multiplying from the left by the block matrix

$$
\left(\begin{array}{cc}
\overline{\mathbf{F}}_{N} & \mathbf{0} \\
\mathbf{0} & \overline{\mathbf{F}}_{N}
\end{array}\right)
$$

and using $\overline{\mathbf{F}}_{N} \mathbf{F}_{N}=\mathbf{I}$ we rewrite the right hand side of (27) as

$$
\left(\begin{array}{cc}
\overline{\mathbf{F}}_{N} & \mathbf{0} \\
\mathbf{0} & \overline{\mathbf{F}}_{N}
\end{array}\right)\left(\begin{array}{c}
\left(\alpha_{s-2 k}\right)_{k=0, s=0}^{N-1,2 N-1} \\
\left(\beta_{s-2 k}\right)_{k=0, s=0}^{N-1,2 N-1}
\end{array}\right) \mathbf{F}_{2 N} \overline{\mathbf{F}}_{2 N} \boldsymbol{\vartheta}
$$

which is nothing else than

$$
\begin{aligned}
& \frac{1}{\sqrt{2 N}}\left(\begin{array}{cc}
\overline{\mathbf{F}}_{N} & \mathbf{0} \\
\mathbf{0} & \overline{\mathbf{F}}_{N}
\end{array}\right)\left(\begin{array}{c}
\left(\hat{\alpha}_{\ell} \mathrm{e}^{-2 \pi \mathrm{i} \ell k / N}\right)_{k=0, s=0}^{N-1,2 N-1} \\
\left(\hat{\beta}_{\ell} \mathrm{e}^{-2 \pi \mathrm{i} \ell k / N}\right)_{k=0, \ell=0}^{N-1,2 N-1}
\end{array}\right) \overline{\mathbf{F}}_{2 N} \boldsymbol{\vartheta} \\
& =\frac{1}{\sqrt{2}}\left(\begin{array}{c|c}
\operatorname{diag}\left(\hat{\alpha}_{k}\right)_{k=0}^{N-1} & \operatorname{diag}\left(\hat{\alpha}_{k+N}\right)_{k=0}^{N-1} \\
\hline \operatorname{diag}\left(\hat{\beta}_{k}\right)_{k=0}^{N-1} & \operatorname{diag}\left(\hat{\beta}_{k+N}\right)_{k=0}^{N-1}
\end{array}\right) \overline{\mathbf{F}}_{2 N} \boldsymbol{\vartheta}
\end{aligned}
$$


Defining the circulant matrices

$$
\begin{gathered}
\mathbf{A}=\mathbf{F}_{N} \operatorname{diag}\left(\hat{\alpha}_{k}\right)_{k=0}^{N-1} \overline{\mathbf{F}}_{N}, \quad \mathbf{B}=\mathbf{F}_{N} \operatorname{diag}\left(\hat{\alpha}_{k}\right)_{k=N}^{2 N-1} \overline{\mathbf{F}}_{N}, \\
\mathbf{C}=\mathbf{F}_{N} \operatorname{diag}\left(\hat{\beta}_{k}\right)_{k=0}^{N-1} \overline{\mathbf{F}}_{N} \quad \text { and } \quad \mathbf{D}=\mathbf{F}_{N} \operatorname{diag}\left(\hat{\beta}_{k}\right)_{k=N}^{2 N-1} \overline{\mathbf{F}}_{N},
\end{gathered}
$$

and using (28) we can rewrite the basis transformation from Theorem 1 as

$$
\left(\begin{array}{c}
\boldsymbol{\varphi} \\
\boldsymbol{\psi}
\end{array}\right)=\frac{1}{\sqrt{2}}\left(\begin{array}{ll}
\mathbf{A} & \mathbf{B} \\
\mathbf{C} & \mathbf{D}
\end{array}\right)\left(\begin{array}{cc}
\mathbf{F}_{N} & \mathbf{0} \\
\mathbf{0} & \mathbf{F}_{N}
\end{array}\right) \overline{\mathbf{F}}_{2 N} \boldsymbol{\vartheta}
$$

Having expressed the transformations as in Theorem 1 it is now straightforward to apply a lifting step or a dual lifting step as described in Definition 1. So one can change directly from arbitrary bases to orthogonal or interpolatory bases. In case of orthogonal bases for $W$ one would change $\boldsymbol{\psi}$ into $\boldsymbol{\psi}^{\perp}$ given by

$$
c_{k}\left(\psi^{\perp}\right)=\frac{c_{k}(\psi)}{\sqrt{N \sum_{p \in \mathbb{Z}}\left|c_{k+N p}(\psi)\right|^{2}}} \quad \text { for all } k \in \mathbb{Z},
$$

and for interpolatory bases with the additional condition

$$
\sum_{p \in \mathbb{Z}} c_{k+N p}(\psi) \neq 0 \quad \text { for all } \quad k=0, \ldots, N-1,
$$

one could change to

$$
c_{k}\left(\psi^{L}\right)=\frac{c_{k}(\psi)}{N \sum_{p \in \mathbb{Z}} c_{k+N p}(\psi)} \quad \text { for all } \quad k \in \mathbb{Z},
$$

obtaining bases with $\psi^{L}\left(\frac{2 k \pi}{N}\right)=\delta_{k, 0}$ for $k=0, \ldots, N-1$. To achieve for example orthogonality of the translates of $\psi$ we can choose $\mathbf{A}=\mathbf{I}, \mathbf{B}=\mathbf{0}$ and we have to replace $\operatorname{diag}\left(\hat{\beta}_{k}\right)_{k=0}^{N-1}$ and $\operatorname{diag}\left(\hat{\beta}_{k}\right)_{k=N}^{2 N-1}$ by

$$
\operatorname{diag}\left(\frac{\hat{\beta}_{k}}{\sum_{p \in \mathbb{Z}} \hat{\beta}_{k+N p} \hat{\vartheta}_{k+N p}}\right)_{k=0}^{N-1} \text { and } \operatorname{diag}\left(\frac{\hat{\beta}_{k+N}}{\sum_{p \in \mathbb{Z}} \hat{\beta}_{k+N p} \hat{\vartheta}_{k+N p}}\right)_{k=0}^{N-1},
$$

respectively.

\section{References}

[CDP96] Carnicer, J.M., Dahmen, W., Pena, J.M.: Local decomposition of refinable spaces and wavelets. Appl. Comput. Harmon. Anal., 3, 127-153 (1996)

[CM93] Chui, C.K., Mhaskar, H.N.: On trigonometric wavelets. Constr. Approx., 9, 167-190 (1993) 
[CDF92] Cohen, A., Daubechies, I., Feauveau, J.-C.: Biorthogonal bases of compactly supported wavelets. Commun. Pure Appl. Math., 45, 485-560 (1992)

[DKU99] Dahmen, W., Kunoth, A., Urban, K.: Biorthogonal spline wavelets on the interval - stability and moment conditions. Appl. Comput. Harmon. Anal., 6, 132-196 (1999)

[DM93] Dahmen, W., Micchelli, C.A.: Banded matrices with banded inverses. II: Locally finite decomposition of spline spaces. Constr. Approx., 9, 263-281 (1993)

[DS99] Dahmen, W., Schneider, R.: Wavelets on manifolds. I: Construction and domain decomposition. SIAM J. Math. Anal., 31, 184-230 (1999)

[DSw98] Daubechies, I., Sweldens, W.: Factoring wavelet transforms into lifting steps. J. Fourier Anal. Appl., 4, 247-269 (1998)

[KLT95] Koh, Y.W., Lee, S.L., Tan, H.H. Periodic orthogonal splines and wavelets. Appl. Comput. Harmonic Anal., 2, 201-218 (1995)

[LMQ01] Lyche, T., Mørken, K., Quak, E.: Theory and algorithms for nonuniform spline wavelets. In: Dyn, N. et al. (ed), Multivariate Approximation and Applications, Cambridge University Press, 152-187 (2001)

[M96] Masson, R.: Biorthogonal spline wavelets on the interval for the resolution of boundary problems. Math. Models Methods Appl. Sci., 6, 749-791 (1996)

[NW96] Narcowich, F.J., Ward, J.D.: Wavelets associated with periodic basis functions. Appl. Comput. Harmon. Anal., 3, 40-56 (1996)

[PT94] Plonka, G., Tasche, M.: A unified approach to periodic wavelets. In: Chui, C.K. et al. (ed), Wavelets: Theory, Algorithms, and Applications, Academic Press, New York, 137-151 (1994)

[PT95] Plonka, G., Tasche, M.: On the computation of periodic spline wavelets. Appl. Comput. Harmon. Anal., 2, 1-14 (1995)

[PQ95] Prestin, J., Quak, E.: Trigonometric interpolation and wavelet decompositions. Num. Alg., 9, 293-317 (1995)

[Q02] Quak, E.: Nonuniform $B$-splines and $B$-wavelets. In: Iske, A. et al. (ed), Tutorials on Multiresolution in Geometric Modelling. Springer, Berlin, 101-146 (2002)

[S98] Schneider, R.: Multiskalen- und Wavelet-Matrixkompression. Analysisbasierte Methoden zur effizienten Lösung großer vollbesetzter Gleichungssysteme. Advances in Numerical Mathematics. B. G. Teubner Stuttgart (1998)

[Se98] Selig, K.: Periodische Wavelet-Packets und eine gradoptimale Schauderbasis. PhD thesis, Universität Rostock, Germany, Shaker Verlag, Aachen (1998)

[SchSw96] Sweldens, W., Schröder, P.: Building your own wavelets at home. Wavelets in Computer Graphics, ACM SIGGRAPH Course Notes (1996)

[Sw96] Sweldens, W.: The lifting scheme: A custom-design construction of biorthogonal wavelets. Appl. Comput. Harmon. Anal., 3, 186-200 (1996) 